\title{
A GSM-MAP Protocol Experiment Using Passive Testing
}

\author{
Marine Tabourier, Ana Cavalli, and Melania Ionescu \\ Institut National des Télécommunications \\ 9, rue Charles Fourier, 91011 Evry Cedex, France \\ \{Marine.Tabourier, Ana.Cavalli, Melania. Ionescu\}@int-evry.fr
}

\begin{abstract}
Passive testing is the process of collecting traces of the messages exchanged between an operating implementation and its environment, in order to verify that these traces actually belong to the language accepted by the provided specification. The specification has in general the form of a finite state machine (FSM). In this paper, we present an extension of the existing algorithms to consider the specification as an extended finite state machine (EFSM). An algorithm is also introduced to take into account the number of states and transitions covered. In this paper we also present the results of our experiments on a signalling protocol that is a component of the European digital cellular radiocommunication system GSM, the GSM-MAP.
\end{abstract}

\section{Introduction}

The objective of conformance testing is to evaluate whether a protocol implementation has the same behaviour as its specification. In active conformance testing, a specification of the behaviour is used to generate test sequences; the implementation under test (IUT) is then placed in a dedicated testing environment, and according to the predefined test sequences, one (or more) tester sends inputs to the IUT, observes the corresponding outputs, and analyses them by comparing them with the expected outputs.

On the other hand, passive testing consists in collecting traces of the messages exchanged between an operating implementation and its environment, and verifying whether these traces actually belong to the language accepted by the specification (in the form of an automaton). In passive testing, there is no test sequence generation, test execution is replaced by simple trace collection, and the main effort is placed on analysis of the observed messages. Though passive testing is sometimes mentioned as an alternative to active testing [6], only little effort has been devoted to this aspect of testing [12, 2. However, we feel that passive testing is worth investigating, since (a) under certain circumstances, it may be the only type of test available, e.g. in network management, (b) it is relatively cheap and easy to implement, and (c) active testing is sometimes impractical due to the complexity of systems.

In this paper, we present the principles of passive testing, and an application to a real protocol, the GSM-MAP. We also extend the algorithm proposed in 
[2] in order to take the state and transition coverage into account and in order to consider an extended finite state machine as the system specification. The SDL specification of the GSM-MAP that we used in our experiment has been obtained from ETSI standard; this specification has been completed in our group [5]. SDL [7] has established itself as the Formal Description Technique of choice in the telecommunication world; for the purpose of conformance testing, the specification is usually considered as an Extended Finite State Machine, the underlying model of SDL.

The paper is organised as follows: in section 2, we present the concepts of passive testing for finite state machines; section 3 presents two algorithms to evaluate test coverage, taking state and transition coverage into account. In section 4 we study the application of this method to real protocols and we introduce an extension of the algorithms in order to consider extended finite state machines as specifications. Section 5 is an application of this method to the GSM-MAP-DSM protocol.

\section{The Basics of Passive Testing}

In passive testing [2], contrary to active testing, the tester does not control the implementation under test. The implementation is in operating condition, and the tester only observes the messages exchanged by the IUT and its environment, in order to check if they correspond to a behaviour compliant with the specification (see Fig. 1).

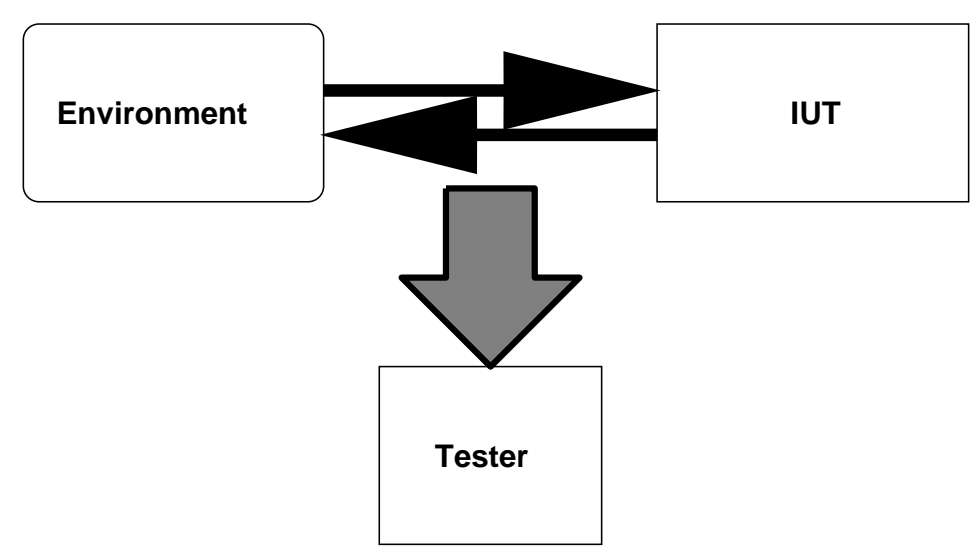

Fig. 1. An architecture for passive testing

The main difficulty in passive testing is that we have no knowledge of the state in which the implementation is at the beginning of the trace. In this respect, passive testing is different from fault diagnostic [4], where the trace is recorded during an active test execution, and the initial state is known. On the 
contrary, in passive testing, no assumption is made about the moment when the recording of trace begins, and therefore it is not necessarily the initial state. Each input/output pair of the trace is assumed to represent a transition in the specification, and our objective is to match the transitions of the trace with those of the specification.

The passive testing process can be decomposed into two steps: the passive homing sequence, in which the current state is found out, and the fault detection phase, in which the trace is compared with the specification.

\subsection{Passive Testing for Deterministic Machines}

First Stage: Passive Homing Sequence. The current state is determined by elimination. Initially, all the states are candidates. The transitions of the trace are studied one after the other: the states which accept the input/output are replaced by the destination state of the corresponding transition (redundant states are eliminated), whereas the states which do not accept it are eliminated. After a number of iterations, there are two possible outcomes:

- either a single state is obtained: it corresponds to the current state, and we proceed to the second stage; or

- an input/output pair that is not accepted by any candidate state is encountered in the trace; it indicates that the behaviour observed does not correspond to the specification, and that a fault has been detected. The algorithm stops.

Second Stage: Fault Detection. From the current state, we follow the trace in the specification. If we reach a state which does not accept the following input/output pair of the trace, there is an error. If not (i.e the end of the trace is reached and there were no deadlocks), no error was detected.

Example. Assume our specification is the FSM in Fig. 2, and the following trace has been observed:

$$
\begin{array}{llllllllll}
\mathrm{a} / 1 & \mathrm{~b} / 2 & \mathrm{c} / 3 & \mathrm{~b} / 2 & \mathrm{~b} / 2 & \mathrm{a} / 1 & \mathrm{~b} / 2 & \mathrm{c} / 3 & \mathrm{c} / 3 & \mathrm{~b} / 3
\end{array}
$$

We can see the evolution of the potential current states (or candidates) during the passive homing sequence, and then the evolution of the actual current state during the fault detection phase (Fig. 3). The first five transitions of the trace allowed us to identify the current state (state 1). The following input/output pairs of the trace are used only to detect faults.

\subsection{Passive Testing for Nondeterministic Machines}

In the case of a nondeterministic specification, the machine can have several possible behaviours for a same input in a given state. If the outputs are different, 


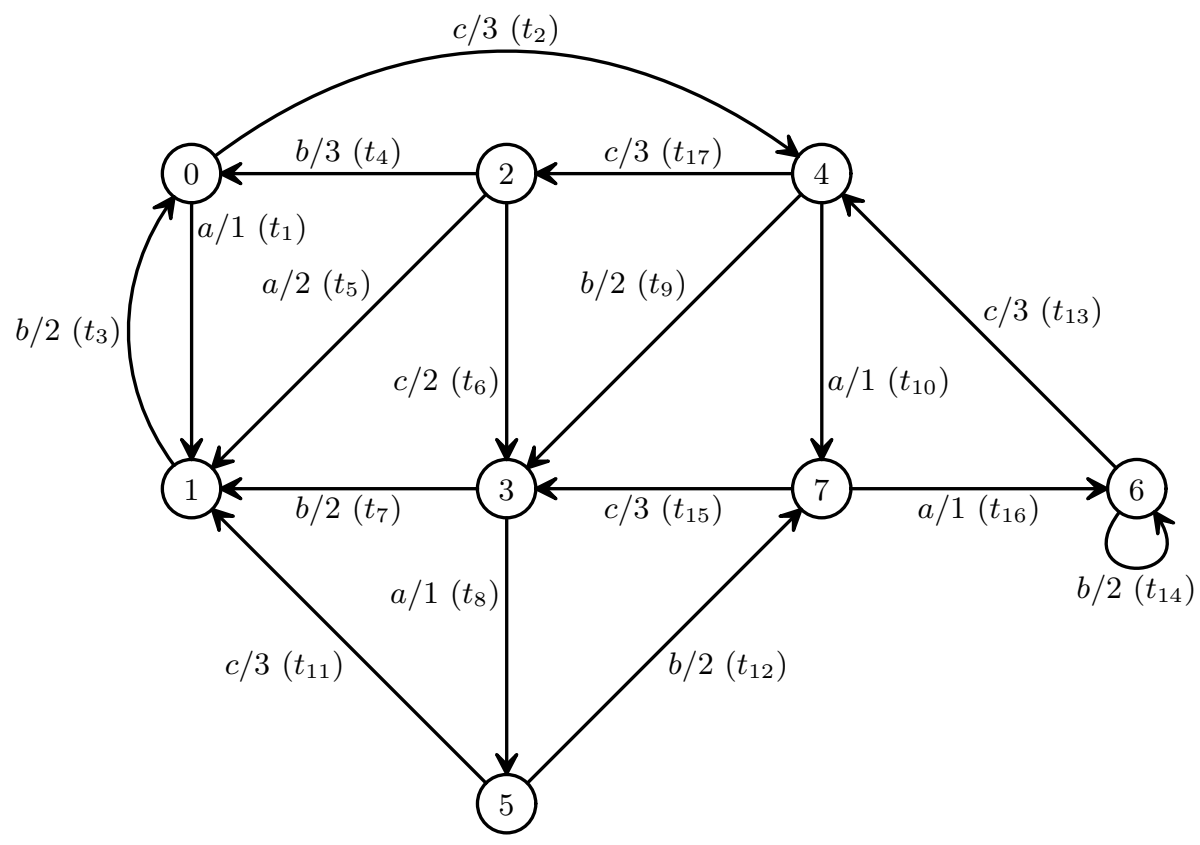

Fig. 2. Example: A deterministic machine

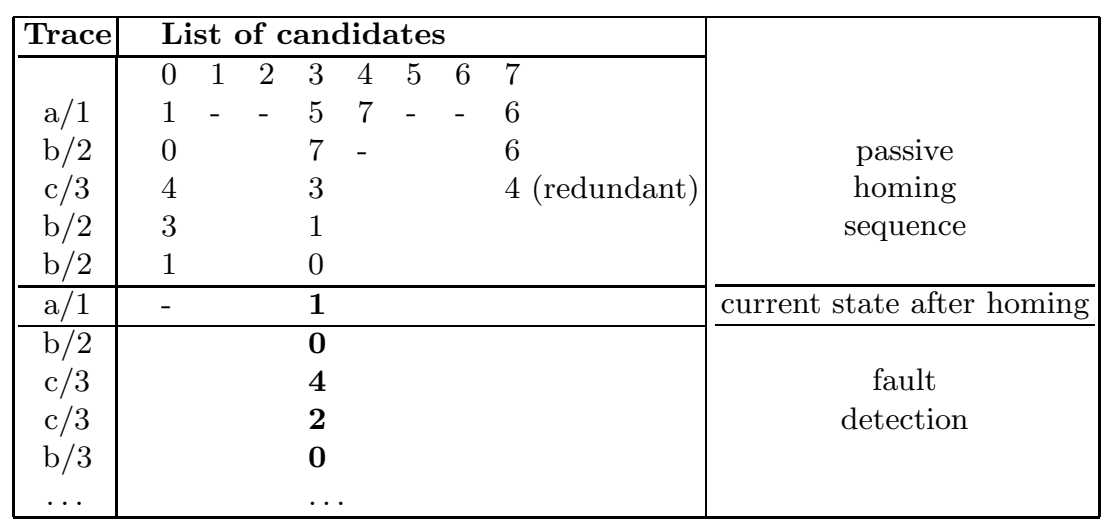

Fig. 3. Candidate list evolution 
this is called observable nondeterminism, otherwise, if the outputs are identical, but the states of arrival are different, it is called unobservable nondeterminism.

In the case of passive testing, observable nondeterminism is not an issue, since the output makes it possible to distinguish between transitions and to know which path was selected; for this reason, the algorithm for passive testing of deterministic machines can also be applied for passive testing of observable nondeterministic machines. On the other hand, this is not possible for unobservable nondeterminism, since the trace is identical for both transitions. Therefore a set of possible states (candidates) has to be maintained: there is no guarantee that this set can be reduced to a single element. Moreover, even if at a point, we do obtain at a singleton, it may, in turn, have several possible successors. Thus there is no actual passive homing procedure, but the principles of the algorithm remain the same.

\section{Test Coverage}

One of the problems with passive testing is that it is impossible to issue a $P A S S$ verdict. Contrary to active testing, we cannot control the implementation under test in order to cover all the transitions. This is due to the fact that the passive tester only records and analyses the IUT's behaviour, but does not originate it. Therefore, even when no fault was detected during the test, it may happen that infrequent parts of the system's behaviour remain untested. Nevertheless, we can try to determine the test coverage, i.e compute the number of states and transitions that have been traversed, and identify which states and which transitions have been traversed and which have not.

\subsection{Test Coverage for Deterministic Machines}

Initial Approach. In the deterministic algorithm, coverage evaluation can be done in the fault detection phase; at this stage, the current state is known, and whenever a state is reached or a transition is fired, we can mark it. Thus we will know which states and transitions have been covered. If all the transitions have been fired, a coverage similar to that of transition tour [10] has been achieved.

If we consider the previous example (Fig. 2), in the fault detection phase (from state 1 on), transitions $t_{3}, t_{2}, t_{17}$, and $t_{4}$ have been fired, which represents a $23 \%$ transition coverage (4 transitions out of 17 ) and a $50 \%$ state coverage (4 states out of 8 ). If the trace was longer (as it obviously happens in real applications), the coverage would be better. For instance, assume our trace continues with:

$$
\begin{array}{cccccc}
\mathrm{c} / 3 & \mathrm{a} / 1 & \mathrm{a} / 1 & \mathrm{~b} / 2 & \mathrm{c} / 3 & \mathrm{~b} / 2
\end{array}
$$

which corresponds to transitions

$$
\begin{array}{llllll}
t_{2} & t_{10} & t_{16} & t_{14} & t_{13} & t_{9}
\end{array}
$$


then 5 additional transitions have been fired, and 3 additional states have been reached, which brings the transition coverage to $53 \%$ and the state coverage to $87 \%$.

Improving the Coverage Evaluation with Backtracking. With the method presented in the previous section, states and transitions are only marked during the fault detection phase. However, if some transitions have been fired in the passive homing sequence, and are not fired again in the fault detection phase, they will not be considered as fired in this evaluation. Thus, the coverage evaluation will be less than the actual coverage. In order to improve this coverage evaluation, we also need to mark the states and transitions that were fired during the first phase. This is done by backtracking the actual states and transitions that have been fired in the passive homing sequence. The algorithms introduced below (algorithm 1 and algorithm 2) are extensions of the algorithm given in [2]. Since we mentioned that we can use the same approach for observable nondeterministic finite state machine as for deterministic finite state machines, we give their formal definition.

Definition 1. An observable nondeterministic finite state machine is a fivetuple $M=\left(S, s_{0}, I, O, \delta\right)$, where

$S$ is a finite nonempty set of states,

$s_{0}$ is the initial state,

$I$ is a finite set of input symbols,

$O$ is a finite set of output symbols, and

$\delta: S \times I \times O \rightarrow S$ is a transition function.

$s^{\prime}=\delta(s, a, o)$ means that there is a transition from $s$ to $s^{\prime}$ labelled with a/o.

Algorithm 1 (Test coverage). This procedure can be inserted either between the passive homing and the fault detection procedures, or after the fault detection procedure, just before computing the coverage (the latter is better in a real-time environment). We denote $\operatorname{Pred}\left(s^{\prime}, a, o\right)$ the set of predecessors of state $s^{\prime}$ for transitions labelled with $a / o: \operatorname{Pred}\left(s^{\prime}, a, o\right)=\left\{s \in S \wedge \delta(s, a, o)=s^{\prime}\right.$.

Input: a specification FSM $S p e c$, the current state $s c u r$, a trace $s=a_{1} / o_{1}, \ldots$, $a_{k} / o_{k}$ reflecting a behaviour of an implementation Imp up to scur.

Output: the set of transitions that were fired in the course of the trace up to scur.

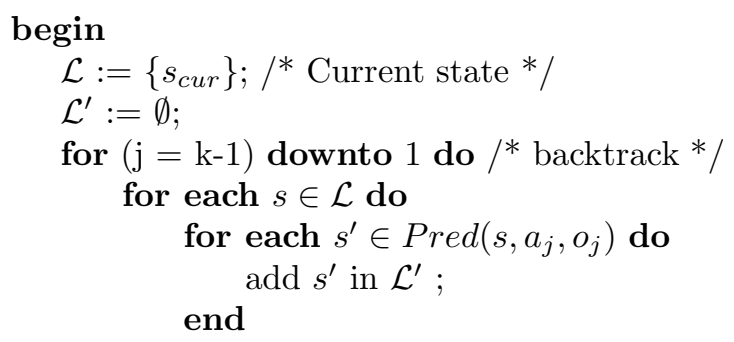




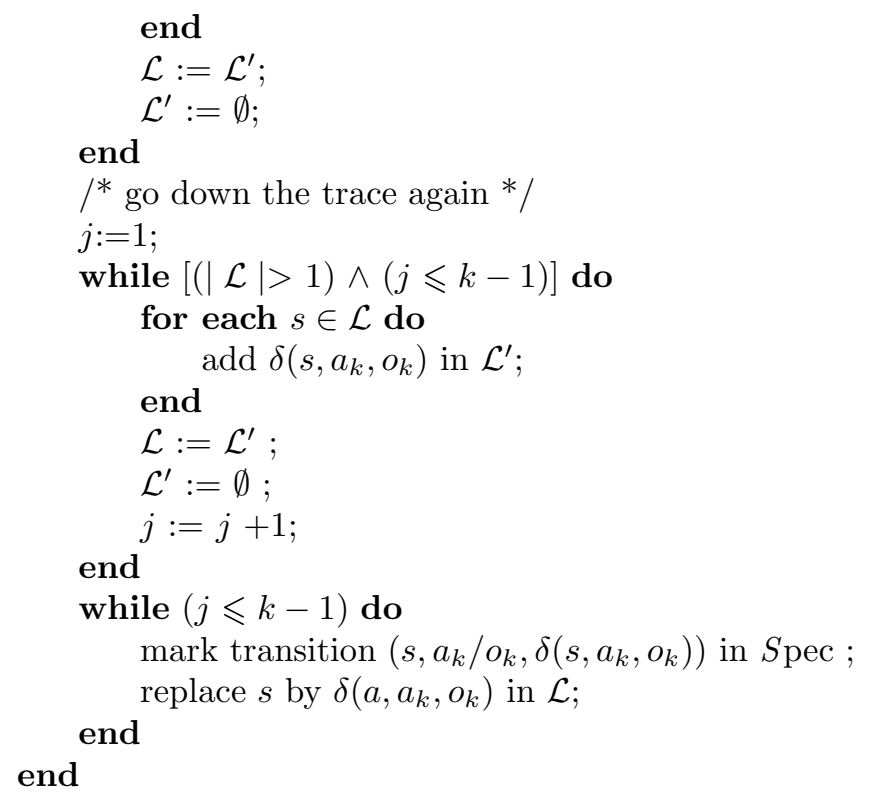

This method will work best if the singleton was obtained because only one candidate accepted the last transition; results will not be as good if the singleton happened because two (or more) paths of the automaton converge towards a single state. This phenomenon is independent of the actual algorithm: if two paths converge during the passive homing sequence, determining which one was actually followed is an undecidable problem. Let us consider two examples to illustrate this:

- Consider again the trace that was used in the example. If we backtrack step by step and then walk down the path again, we can find the transitions that were fired during the passive homing sequence, namely transitions $t_{8}, t_{12}$, $t_{15}, t_{7}, t_{3}$ and $t_{1}$. If we take them into account to evaluate the coverage, 14 different transitions have been fired, which makes for a $82 \%$ transition coverage and a 100\% state coverage. In this case, the backtracking procedure has allowed us to improve the coverage significantly.

- On the other hand, let us now assume that the trace had begun with

$$
\mathrm{b} / 2 \mathrm{c} / 3 \quad \mathrm{~b} / 2 \mathrm{a} / 1
$$

for the same FSM. Then, the passive homing sequence shows that the current state is state 5 after 4 transitions. With backtracking, we can find out that the last two transitions were transitions $t_{9}$ followed by transition $t_{8}$, but we cannot determine whether the initial state was state 6 , with transitions $t_{14}$ and $t_{13}$, or state 1 with transitions $t_{3}$ and $t_{2}$, as both paths converge towards state 5 . In this example, despite the convergence, backtracking has allowed us to mark two transitions and two states. 


\subsection{Test Coverage for Unobservable Nondeterministic Machines}

For an unobservable nondeterministic finite state machine (NDFSM), it is more difficult to mark the transitions that are fired, as we do not know a priori which of the candidate transition has been fired. However, we can still use the approach above and mark the transitions whenever the set of candidates is a singleton, and stop marking when a (unobservable) nondeterministic transition is encountered. This method will give us a lower bound of the actual coverage.

The formal definition for an unobservable NDFSM is the same as Definition, 1 , except $\delta$ is a transition relation instead of a function. Our definition of the set of predecessors Pred is modified accordingly:

If $|\delta(s, a, o)|=1$, then $\operatorname{succ}(s, a, o)$ denotes its unique element.

Algorithm 2 (Coverage for a nondeterministic machine). Input: an NDFSM Spec (specification) and an input/output trace reflecting a behaviour of implementation under test Imp.

Output: whether the trace from $\operatorname{Imp}$ is accepted by $S p e c$, with an evaluation of transition coverage.

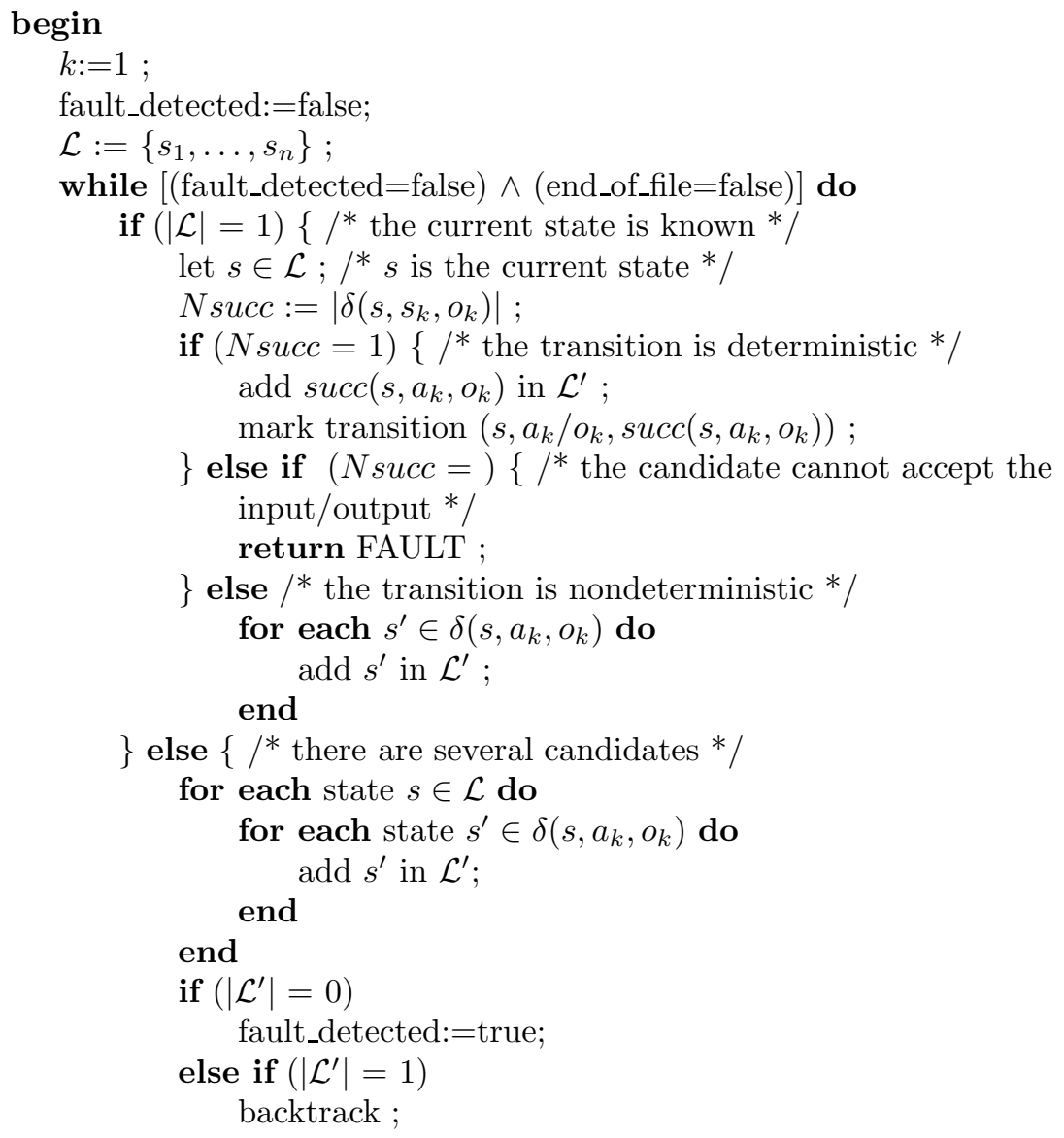




$$
\begin{aligned}
& \} \\
& \mathcal{L}:=\mathcal{L}^{\prime} \\
& \mathcal{L}^{\prime}:=\emptyset \text {; } \\
& k:=k+1 \text {; } \\
& \text { end } \\
& \text { if (fault_detected) } \\
& \text { return FAULT; }
\end{aligned}
$$

\section{backtrack}

$j:=k-1$;

while $\left[(j>0) \wedge\left(\left|\operatorname{Pred}\left(s, a_{j}, o_{j}\right)\right|=1\right)\right]$ do

mark transition $\left.\left(\operatorname{pred}\left(s, a_{j}, o_{j}\right), a_{j} / o_{j}, s\right)\right)$;

end

$s:=\operatorname{pred}\left(s, a_{j}, o_{j}\right)$;

$j:=j-1$

With state and transition coverage, passive testing can be used primarily when it is impossible to control the implementation, but also as a complement of active testing. Indeed, as protocols are getting more and more complex, test sequences are getting increasingly long and sometimes impossible to generate and execute in a reasonable period of time. Therefore, a possible way to alleviate this problem is to generate and execute test sequences only for the core behaviour of a protocol (or for its critical functionalities), then to test it passively. Then, after studying both the active and passive test coverage, new active test sequences can be produced and executed for the transitions that have not been tested (if any).

\section{Application to Real Protocols}

\subsection{Extended Finite State Machine}

Real life protocols are too complex to be modelled with a simple Finite State Machine. In order to specify a real protocol, we must use the Extended Finite State Machine (EFSM) formalism (i.e there will be internal variables and parameters). There may be a predicate on variables associated with each transition of an EFSM. The predicate must be true for the transition to be fireable. A transition may also have actions on the variables, modifying their values. In this paper, we consider only normalized EFSM, which are composed of a unique module, and whose actions do not include complex instructions (IF-THEN-ELSE, WHILE, etc.). An EFSM can always be transformed into a normalized EFSM [1]. We define it formally:

Definition 2. An extended finite state machine is a six-tuple $M=\left(S, s_{0}, I, O\right.$, $\boldsymbol{x}, T)$ where $S$ is a finite nonempty set of states, $s_{0}$ is the initial state, $I$ is a finite set of input symbols, $O$ is a finite set of output symbols, $\boldsymbol{x}$ is a vector denoting a finite set of variables, $T$ is a finite set of transitions. 
Each transition $t$ in $T$ is a six-tuple $t=\left(s_{t}, f_{t}, i_{t}, o_{t}, P_{t}, A_{t}\right)$ where $s_{t}$ is the transition starting state, $f_{t}$ is the transition ending state, $i_{t}$ is the input, $o_{t}$ is the output, $P_{t}$ is a predicate on the current values of the variables $\left(P_{t}(\boldsymbol{x})\right.$ is TRUE or FALSE) $A_{t}$ is an action on variables values $\left(\boldsymbol{x}:=A_{t}(\boldsymbol{x})\right)$.

Definition 3. We define a parametrised extended finite state machine as a generalisation of Definition 2, where an input or output symbol has a set of (zero or more) parameters $\boldsymbol{y}=\left(y_{1}, \ldots, y_{p}\right)$. For a transition $t$, the input, output, predicate and action all depend on $\boldsymbol{y}: i_{t}(\boldsymbol{y}), o_{t}(\boldsymbol{y}), P_{t}(\boldsymbol{y})$ and $\boldsymbol{x}:=A_{t}(\boldsymbol{x}, \boldsymbol{y})$.

The use of extended finite states machines raises a new issue: how should we handle these variables? In active testing, it is easy to know the value of the variables, because the initial state is known. In passive testing, we do not know their value, since the machine can be in any state at the beginning of the trace. We propose several approaches:

- generate the reachability graph;

- transform the extended finite state machine into a nondeterministic finite state machine;

- determine the variable values.

We will study these strategies in the following sections and we will point out their advantages and their weaknesses.

\subsection{Reachability Graph}

The simplest approach is to generate the reachability graph, which is tantamount to unfolding the EFSM to its equivalent FSM. Unfortunately, this causes the well-known state explosion problem.

However, we can cope with this problem if we experiment this approach on a protocol that is not too complex (see Sect. 5). We use verification techniques to alleviate some of the problems and reduce the size of the generated reachability graph [1. The reachability graph we obtain is an unfolding of the EFSM specification, which includes instantiations of the variables and parameters used in the original specification.

\subsection{Transforming the EFSM into an NDFSM}

Basically, the EFSM can be transformed into an NDFSM by removing the predicates enabling the transitions. If we do this transformation, following the example in Fig.4, we can then simply apply the algorithm for nondeterministic machines.

With this method, the detection power is lessened. Faults caused by bad parameters values or by control variables will not be detected. In the first example on Fig. 4 (observable case), both $a / b$ and $a / c$ would be accepted, whereas only one of them is allowed in the original specification; in the nonobservable case, both $S_{j}$ ou $S_{k}$ would be accepted as the final state. Since the accepted machines 

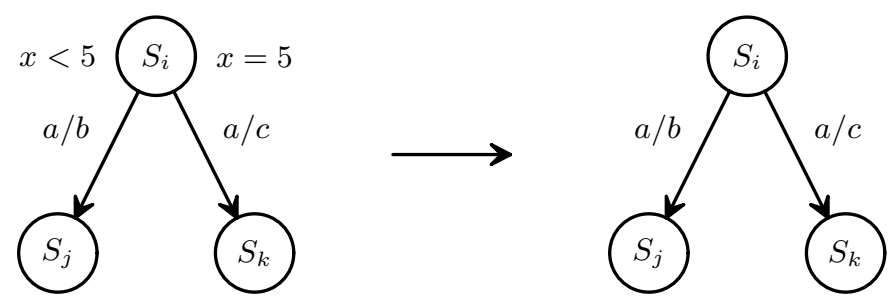

Observable
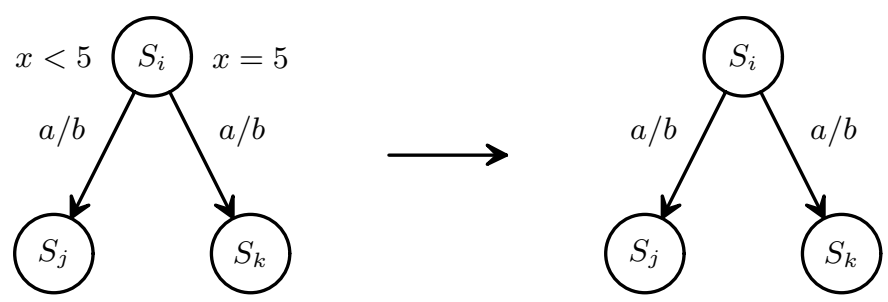

Non - observable

Fig. 4. Transforming an EFSM into an ND-FSM

are still a superset of the correct machines, this solution is acceptable, as no correct implementation will be rejected. However, when using this approach, one must be aware that a faulty implementation may be accepted, and, more specifically, a behaviour that is accepted by the nondeterministic machine may be incorrect in the original specification.

In conclusion, this approach allows us to detect glaring mistakes rapidly, avoiding the state explosion problem. But more precise testing will still be necessary to ensure conformance.

\subsection{Determining Variable Values}

Principle. With the extended finite state machine formalism, the variable values can sometimes be deduced from the input/output trace. For instance, consider the situation depicted in Fig. 5. where there are two possible transitions from state $S_{i}$ upon receipt of input $a$, depending on the current value of variable $x$. Assume that we know the current state is $S_{i}$, but that the current value of variable $x$ is unknown. If the next input/output pair in the trace is "a/1", we can deduce that, after the transition, the machine will be in state $S_{k}$, and also that variable $x$ will have the value 0 . Therefore we propose to reuse the idea of the section 4.3: predicates depending on variables of unknown value are not taken into account, making the transition temporarily nondeterministic. However, as soon as the value of a variable has been determined, this value will be used 
to evaluate the predicates, and will possibly be modified depending on actions. With this approach, a transition is fireable if:

- the input/output of the trace matches the label of the transition; and

- either the predicate is true, or it cannot be evaluated (due to unknown variable values).

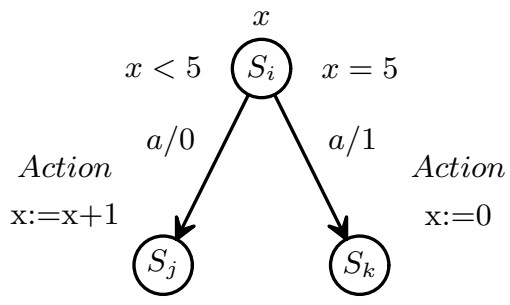

Fig. 5. Finding out variables values

Loss of Values Problem Our goal with this approach is to be able to ultimately determine all the variable values, which will allow us to actually evaluate all the predicates, and reject a transition if its predicate is false. However, note that as long as some variables have unknown values, there is a risk that, for some transitions, variables whose values had already been determined may become unknown again. We illustrate this "loss of value" problem in Fig. 6] In this example, we assume that the value of variable $x$ has already been determined $(x=3)$ and that the value of $y$ is still unknown. Assume that $y<5$; then the left branch is fired, and after the transition, $x=0$ holds; on the contrary, if we now assume that $y=5$, then the right branch is fired, and after the transition, we have $x=3$ and $y=5$. Since the observable behaviour is the same for both branches ("a/0"), we cannot determine, from the trace, which branch was selected, and both $x$ and $y$ are unknown after the transition. Of course, once all the variables are known, the loss of value problem cannot occur anymore.

Algorithm Variables that have not been determined are characterized by a special value UNDEFINED. Since the extended FSM is deterministic, there will be a passive homing phase (trying to determine both the current state and all the variable values), followed by a fault detection phase (checking whether the input/output that are observed are conformant to the specification, taking all the predicates and actions into account - i.e. checking whether they are accepted by the automaton for the current state and values). The rules for changing the variable values in the passive homing phase are as follows:

- A transition can determine a variable either if the variable appears at the left-hand side of an assignment or if it appears in the transition predicate. 


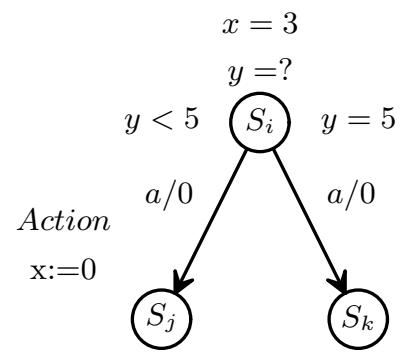

Fig. 6. Loss of variable information

- For a given input/output pair, if there are several candidate transitions that might determine a variable, but they would give it different values, the variable stays UNDEFINED.

- If an UNDEFINED variable appears in a predicate, the predicate is considered nondeterministic (the branch is accepted).

- If there is a loss of value for a variable, it becomes UNDEFINED again.

\section{Algorithm 3 (Passive testing of an EFSM).}

The transition function $\delta$ is modified to take the predicate into account. $s^{\prime}=\delta(s, a, o, P)$ means that there is a transition from state $s$ to state $s^{\prime}$ labelled "a/o" and guarded by predicate $P$.

Input: A specification EFSM Spec and a trace s of a behaviour of an implementation Imp.

Output: whether the trace from Imp belongs to the language of Spec.

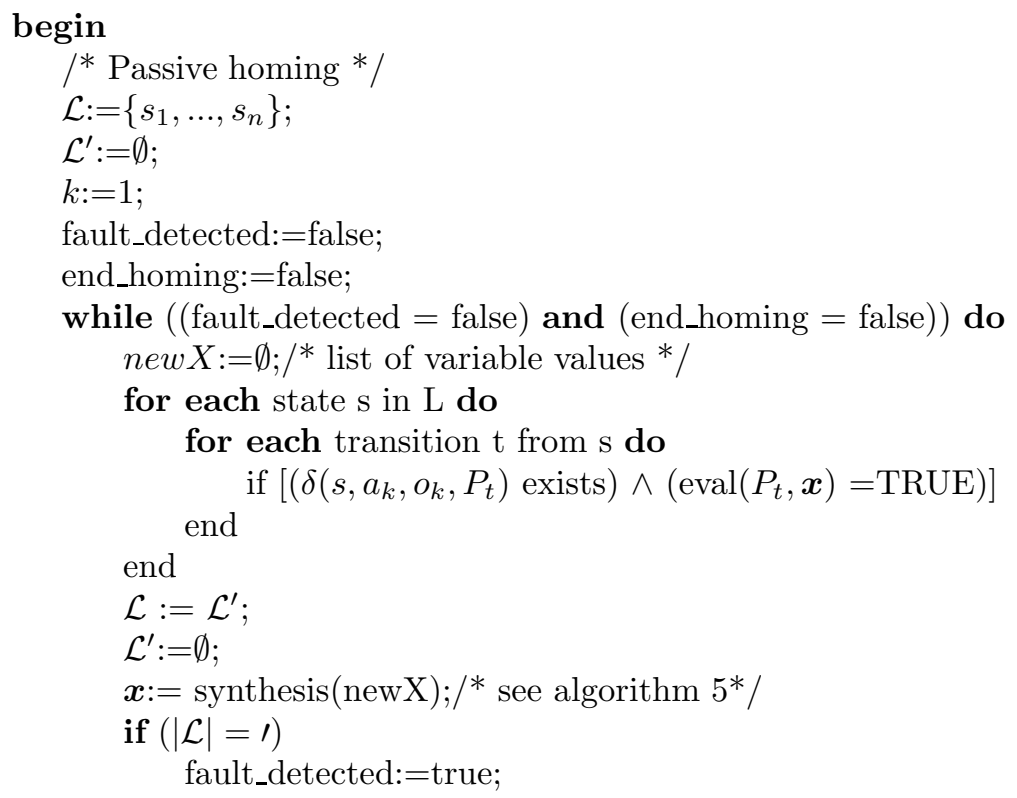




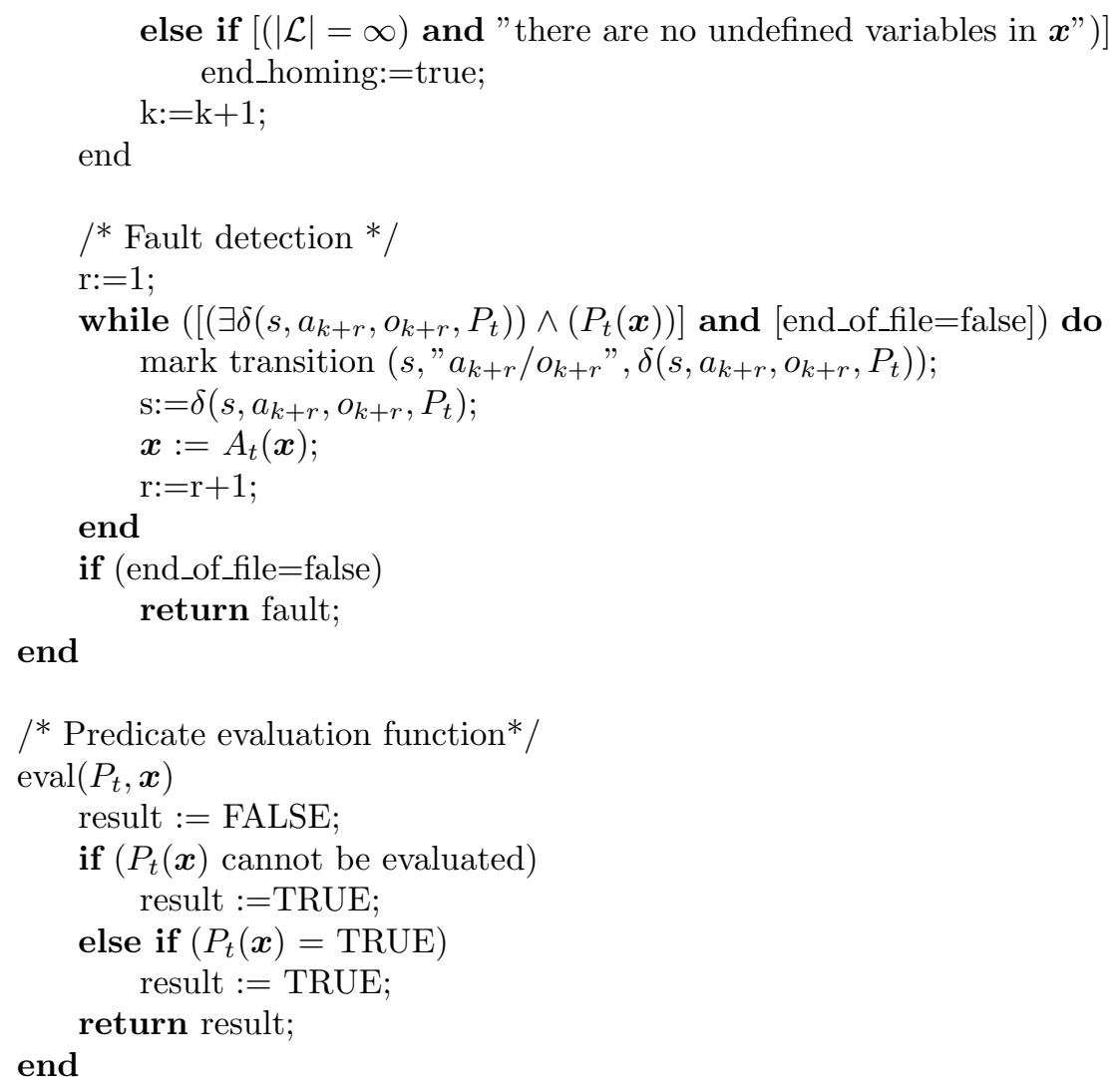

Algorithm 4 (procedure: Adding $A(x)$ to newX list).

begin

$\boldsymbol{y}:=\boldsymbol{x}$

For each component $y_{i}$ of $\boldsymbol{y}$ do

if $\left[\left(x_{i}=\mathrm{UNDEFINED}\right)\right.$ and (a clause of the predicate is $\left.\left.\left(x_{i}=\mathrm{val}\right)\right)\right]$

end $y_{i}:=$ val;

$\boldsymbol{y}:=A(\boldsymbol{y})$

Add $\boldsymbol{y}$ to the list newX;

end

Algorithm 5 (procedure: Synthesis of possible values).

begin

for each variable $x_{i}$

read the list of variables $x_{i}$ 


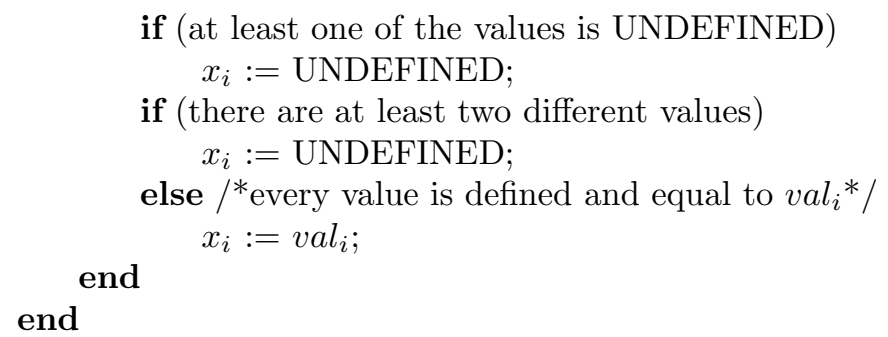

\section{An Experiment with the GSM-MAP Protocol}

In this section we present an application of the passive testing tool that implements the algorithms described in the previous section. The application is the testing in isolation for the MAP-DSM process (MAP-Dialogue State Machine). MAP-DSM is a component process of the GSM-MAP protocol (Global System for Mobile communication-Mobile Application Part) [3]. In order to understand this application we provide a short description of the GSM-MAP protocol and the new approach for the SDL modelling of the MAP-DSM process. Finally, we describe the results obtained using the passive testing tool for the random simulations of this process.

\subsection{GSM-MAP Protocol}

The GSM-MAP protocol describes the signalling functions required in the SS7 signalling system in order to offer the services needed in a mobile network. The machine associated with the GSM-MAP protocol provides the set of services SSM (Service State Machine), for which we distinguish the requesting and the performing services. All these services need the establishment of a dialogue between the requesting and performing entities, dialogue which is coordinated by the MAP-DSM process. This process is responsible for the establishment of the dialogue, transmission and reception of messages, data verification and verification that the context does not change during the dialogue.

\subsection{A New Approach for the SDL Modelling of the MAP-DSM Process}

It must be mentioned here that we have already performed some researches for the testing of the MAP-DSM process [1], but because of its complexity these tests were done only for a partial behaviour of this process. Our original approach for the SDL modelling of the MAP-DSM process intends to reduce the complexity of this component in order to be able to test its global behaviour.

The modelisation of the MAP-DSM process has been performed in the SDL language. The SDL language [7] is a formal description technique, standardized by ITU (International Telecommunication Union). SDL is largely used in the telecommunication industry to make the formal specification of communication 
protocols. By using SDL we have completed the quasi formal specification of the MAP-DSM process [5], which is provided by ETSI standard of the GSMMAP protocol 3. The SDL specification of the MAP-DSM process we have performed represents the complete behaviour of the process. This specification posseses: 2,100 lines of the textual form of the SDL language, 8 main states with 20 internal variables and 135 transition branches.

Our testing tool TESTGEN-SDL [1, 9] uses the accessibility graph obtained by the exhaustive simulation [13] of the SDL specification of a system. The accessibility graph for the MAP-DSM process has 156,996 states and 2,803,651 transitions, with a coverage of $100 \%$ states and $98.31 \%$ transitions. There is only the "discard" transition that was not fired, which means that there are not unexpected messages in the SDL specification.

Because of the huge number of states and transitions it was not possible to test this process using the usual testing methods and we have used for our research an automaton that covers all the states and all the transitions characterizing the behaviour of the process, but which does not contain all the possible scenarios. Finally, this automaton had 3,790 states and 47,478 transitions, with the same coverage of states and transitions as the initial huge one.

But if we want to test the global behaviour of this process, we must be able to test the initial huge automaton. And more than that, we must even use a larger range for the values of the parameters of the input messages in the files used to start the simulation, in order to be closer to the real behaviour of the GSM-MAP protocol.

Using a larger range for the values of the parameters of the input messages, the generated global reachability graph was bigger: 275,042 states and 7,482,243 transitions, with the coverage of $100 \%$ states and $97.96 \%$ transitions and the complexity of the obtained automaton makes impossible its global behaviour's testing.

Knowing that MAP-DSM process uses two complex procedures, twice each one, in order to be able to test the global behaviour of this process, we have used a new original approach of SDL modelling of procedures. Until now researchers substitute the procedure calls by some additional states created in the SDL specification of processes [5].

The new approach we propose is to model the behaviour of a procedure as a process itself and to introduce internal signals in order to model the messages exchanged between the main process and the procedures (see Fig. 7). After that, we have applied our testing tool TESTGEN-SDL [1, 9] in the following way:

- using the decomposition method of processes (see Fig. 7) assuming that the processes exchange messages with the environment,

- using reduction algorithms for each process of the system,

- using a composition algorithm to compose the reduced automata and to obtain the minimized global behaviour of the initial system. By composition, the internal signals introduced in order to exchange messages between the processes were eliminated. 


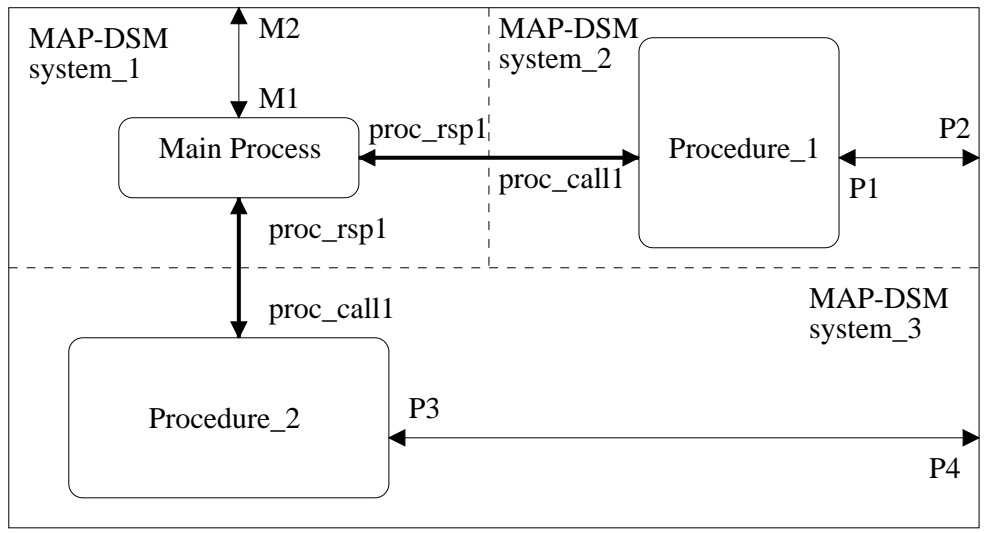

proc_call1, proc_rsp1, proc_call2, proc_rsp2: internal signals introduced between the main process and the procedures

M1, M2: lists of signals exchanged between the main process and the environment P1, P2, P3, P4: lists of signals exchanged between the procedures and environment MAP-DSM system_1, system_2, system_3: systems obtained by the decomposition of the MAP-DSM process

Fig. 7. SDL modelling for the MAP-DSM process

Detailed information on the size of the various machines, both in SDL form and as finite state machines after minimization, is represented in Table 1.

It must be noted that this new approach of the SDL modelling of procedures gives us the possibility to test the complete behaviour of the MAP-DSM process. It allows us even to use a larger range for the values of the parameters of the input messages of the SDL specification and to overcome the automata's explosion, because the final composed automaton associated to the global behaviour of the process has 60 states and 2,136 transitions, or the initial accessibility graph has 275,042 states and 7,482,243 transitions. More than that, our testing tool TESTGEN-SDL allows a totally automatic test generation.

To validate this new approach, we have applied passive testing to this system. The idea is to verify if the system obtained by composition has the same trace as the initial global system. To perform this verification we used the passive testing tool developed in our laboratory. The global SDL specification was also used in this experiment, as we will explain in section 5.3

Note that our specification is actually nondeterministic, as some parts of the behaviour were modelled with SDL informal decisions. However, all the occurrences of nondeterminism are observable, which makes it possible to use the algorithm for deterministic machines, as explained in section 2.2. 


\begin{tabular}{|l|c|c|c|c|}
\hline & main & proc1 & proc2 & composed automaton \\
\hline main states (SDL) & 8 & 2 & 2 & \\
\hline transition branches (SDL) & 46 & 25 & 25 & \\
\hline internal variables (SDL) & 21 & 21 & 21 & \\
\hline states (reachability graph) & 3362 & 3446 & 2738 & \\
\hline transitions (reachability graph) & 17475 & 100421 & 97205 & \\
\hline states (minimized machine) & 24 & 6 & 6 & 60 \\
\hline transitions (minimized machine) & 176 & 352 & 316 & 2136 \\
\hline
\end{tabular}

Table 1. Information on the size of automata

\subsection{MSC Traces}

The global MAP-DSM specification in SDL was used to obtain traces: more specifically, random simulations of the specification were generated using Verilog's ObjectGEODE Simulator [13] and saved as Message Sequence Charts (MSCs) 8] in order to obtain traces conformant to the specification (Fig. 8). These MSCs were then translated into our trace format before being processed by the passive testing tool (Fig. (9).

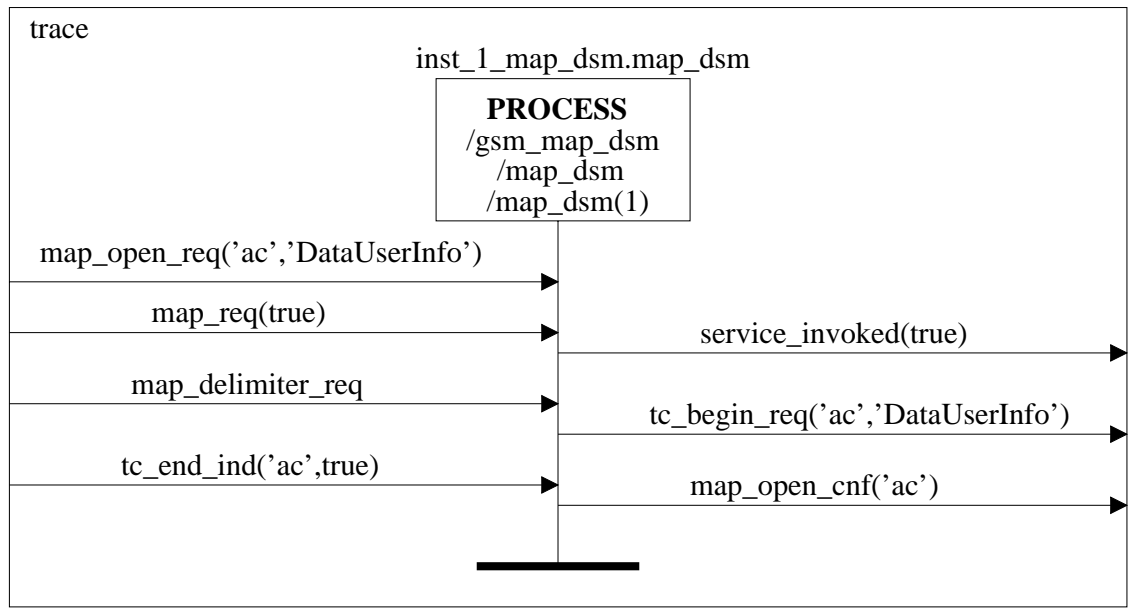

Fig. 8. A sample MSC example

Our passive testing tool uses traces in the following format: each line represents an input/output pair as a quoted string " $i / o$ ", where $i$ is a (possibly parametrised) signal and $o$ is a sequence of 1 or more (possibly parametrised) signals. A NULL signal as output means that no output was observed. Parameters are included within square brackets. "map_req[true]/service_invoked[true]" 


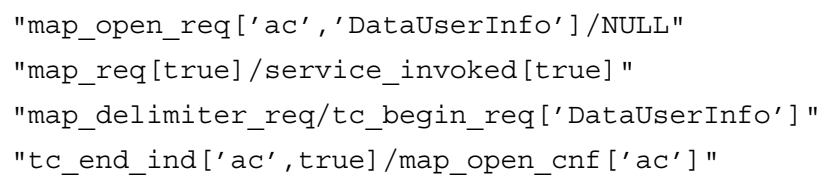

Fig. 9. MSC translated into trace format

means that the system received the signal map_req with the parameter value true as and responded with the signal service_invoked with the parameter value true.

\subsection{Results Obtained}

The first results were the detection of discrepancies between the global SDL specification and the behaviour of the FSM obtained through the decomposition/composition process. These discrepancies were detected within 10 input/output pairs after the end of the passive homing sequence and were reported as faults by the passive testing software. They turned out to be mostly due to some variable values that were inconsistent between the various processes. This allowed us to correct these inconsistencies in the specification. After correction, tests were rerun with the same traces, and no faults were detected, which was the expected outcome. In order to assess our tool, faults were also added manually in some of the traces. Those faults were detected within a few input/output pairs after they occurred.

Traces varied from 1,000 to about 12,500 input/output pairs. Computation time varied between 0.04 to 0.14 seconds on a Sun Ultra1. Transition coverage varied between $5 \%$ and $25 \%$ of the transitions, and state coverage varied between $56 \%$ and $71 \%$ of the states. The global coverage, obtained by taking into account all the states reached in the various experiments, and all the transitions, was $86 \%$ of the states and $46 \%$ of the transitions.

\section{Conclusion and Future Work}

In this paper, we have applied passive testing techniques to a real protocol, the GSM-MAP. The experiments we performed showed that passive testing is a promising technique. It can be applied to test systems where active testing is hard to perform or where no control is possible over the system to be tested. It can also be very useful to improve the specification used as a reference. In fact, some of the discrepancies we found during the experiments were produced by ambiguities in the specification used as a reference rather than by errors of the observed system.

In order to assess the passive testing algorithm and its coverage evaluation, random simulations of the SDL specification of the protocol were saved as MSCs, representing execution traces. A number of faults were detected during our experiments. These faults reflected real differences between the global 
SDL execution traces and the behaviour of the EFSM obtained through the decomposition/composition process.

The EFSM we considered for our experiments was in the form of a reachability graph obtained by the exhaustive simulation of the SDL specification of the system. Our future work will be on the application of our methods directly on an EFSM specification.

\section{Acknowledgements}

We are indebted to David Lee, who helped us with very useful discussions on this subject.

\section{References}

[1] R. Anido, A. Cavalli, T. Macavei, L. Paula Lima Jr, M. Clatin, and M. Phalippou. Testing a real protocol with the aid of verification techniques. In XXII SEMISH, Brazil, August 1996.

[2] David Lee et al. Passive testing and applications to network management. In ICNP'97 International Conference on Network Protocols, Atlanta, Georgia, October 1997.

[3] ETSI. Digital cellular telecommunication system (Phase 2+); Mobile Application Part (MAP) specification (GSM 09.02). ETSI, August 1996.

[4] A. Ghedamsi, R. Dssouli, and G.v. Bochmann. Diagnostic tests for single transition faults in non-deterministic finite state machines. In IWPTS'92 International Workshop on Protocol Test Systems, September 1992.

[5] Melania Ionescu and Ana Cavalli. Test imbriqu du protocole GSM-MAP. In CFIP'99, Colloque Francophone sur l'Ingnierie des protocoles, Nancy, France, April 1999.

[6] ISO and ITU-T. Open distributed Processing - Reference Model - Part 2: Foundations. International Standard 10746-2/ITU-T Recommendation X.902. ITU-T, 1995.

[7] ITU. Recommendation Z.100: Specification and Description Language (SDL). International Standard Z.100. ITU, Geneva, 1993.

[8] ITU-T. Recommendation Z.120: Message Sequence Chart. International Standard Z.120. ITU-T, 1994.

[9] L. Paula Lima and A. Cavalli. A pragmatic approach to generating test sequences for embedded systems. In Proceedings of the IFIP IWTCS'97, Cheju Island, Korea, September 1997.

[10] S. Naito and M. Tsunoyama. Fault detection for sequential machines by transition tour. In Proc. IEEE Computer Conference, 1981.

[11] B. Sarikaya and G. v. Bochmann. Obtaining normal form specifications for protocols. In Computer Network Usage: Recent Experiences. Elsevier Science Publishers, 1986.

[12] Charles L. Seitz. An approach to designing checking experiments based on a dynamic model. In Z. Kohavi and A. Paz, editors, Theory of Machines and Computations. Academic Press, 1972.

[13] Verilog. ObjectGEODE SDL Simulator - Reference manual, 1997. 